\title{
Editorial
}

\section{Structural Biology of Proteins}

Proteins are one of the most versatile biomolecules and they perform diverse functions in living organisms. The function of a protein is mainly dictated by its structure and understanding the structure-function relationship of proteins is a challenging task. The structural biology of proteins aids to understand the structure-function relationship, such as the three-dimensional structure determination of proteins and their complexes, structural analysis of proteins, molecular dynamics simulations, protein folding rates, protein stability and protein interactions. This special issue on "Structural biology of proteins" addresses most of these aspects with latest developments.

Kumarevel revealed the anti-termination mechanism of proteins through structural and functional analysis of a protein-RNA complex, HutP. Sudandiradoss and Sethumadhavan performed molecular docking simulations of scorpin toxins and human voltage-gated potassium channel and identified the key residues for binding. The influence of disulfide bonds in antifungal proteins and peptides has been reported with molecular dynamics simulations by Jayanthi et al. Saravanan and Selvaraj analyzed the long-range contacts and networks in homologous family of proteins. Gromiha showed the intrinsic relationship of amino acid composition/occurrence with topological parameters and folding rates. The relative contributions of strong and weak interactions in epidermal growth factor receptor tyrosine kinase have been evaluated by Swarnadeepa et al. Huang et al. developed a fuzzy query method with human readable rules for predicting the stability of proteins upon amino acid replacements. Further, a novel method has been proposed for predicting the GPCR-G protein coupling selectivity using amino acid properties by Yabuki et al.

In essence, this special issue comprehends the exciting developments in the area of structural biology of proteins and it will be a valuable resource for computational biologists, biochemists, biophysicists, bioinformaticians and researchers working in the field of proteins.

Finally, I would like to thank all the authors for their contributions and cooperation to complete the task.

\section{Michael Gromiha}

Computational Biology Research Center (CBRC), National Institute of Advanced Industrial Science and Technology (AIST), AIST Tokyo Waterfront Bio-IT Research Building, 2-42 Aomi, Koto-ku, Tokyo 135-0064, Japan;

Tel: +81-3-3599-8046;

Fax: +81-3-3599-8081; E-mail: michael-gromiha@aist.go.jp 